\title{
O Uso do GeoGebra e Impressora 3D como Recurso Didático para o Ensino da Geometria das Coordenadas
}

\author{
Rayane Luzia de Andrade Batista ${ }^{1}$, Jarles Tarsso Gomes Santos ${ }^{2}$
}
${ }^{1}$ Curso Técnico em Informática - ECIT Padre Jerônimo Lauwen - Santa Luzia - PB
2Programa de Pós-graduação em Inovação em Tecnologias Educacionais Universidade Federal do Rio Grande do Norte (UFRN) - Natal - RN
\{luzia.rayane15,jarlestarsso\} @gmail.com

\begin{abstract}
The present paper aims to present a possibility of using new technologies in the classroom, through an activity developed with a mathematics teacher, addressing the geometry content of the coordinates, in a class of the 3rd grade of high school, in a Brazilian school. In the example shown, a 3D printer and the GeoGebra software were used, seeking to develop and investigate three-dimensional models, used in the classroom by the teacher during class. From observation and interview with the teacher, after the class, it was possible to verify that the use of these tools can facilitate the visualization and understanding of mathematical concepts, which until then could only be seen in $2 D$.
\end{abstract}

Resumo. O presente trabalho tem como objetivo mostrar uma possibilidade de uso das novas tecnologias em sala de aula, por meio de uma atividade desenvolvida junto a um professor de matemática, abordando o conteúdo de geometria das coordenadas, em uma turma da $3^{a}$ série do ensino médio. $\mathrm{Na}$ ocasião, foram utilizados uma impressora $3 D$ e o software GeoGebra, buscando desenvolver e investigar modelos tridimensionais, usados em sala pelo professor durante a execução da aula. A partir de observações e entrevista com o professor, após a aula, foi possível constatar que o uso dessas ferramentas pode facilitar a visualização e compreensão de conceitos matemáticos, que até então podiam ser vistos apenas em $2 D$.

\section{Introdução}

Atualmente, a tecnologia encontra-se presente nos mais diversos campos da sociedade, aprimorando a forma como agimos e nos comunicamos com as outras pessoas. De acordo com Bortolini et al. (2012), a tecnologia tem proporcionado diferentes formas de realizarmos atividades cotidianas e tem mudado constantemente o modo como nos relacionamos com o mundo à nossa volta. Ainda segundo o autor, essa tecnologia contribui para intensificar as diferentes formas de aprendizagem.

Presente nos mais diversos meios, a tecnologia não se faz diferente na educação. A cada dia precisamos nos adaptar às diversas transformações dos recursos tecnológicos que a contemporaneidade nos reivindica. Nas instituições de ensino, por exemplo, esse avanço tecnológico tem exigido que as gestões escolares tomem posicionamento sobre essa realidade [Koch 2013]. 
Para Stinghen (2016) a tecnologia vem sendo renovada a cada dia, e é de extrema importância que os professores conheçam as possibilidades de utilizar esses recursos tecnológicos a seu favor, e usá-los como ferramenta de aprendizagem em seu cotidiano, mesmo que seja uma tarefa árdua na realidade das escolas públicas.

Hoje são vastas as possibilidades de recursos tecnológicos que podem ser utilizados para melhorar o processo de ensino. Segundo Almeida e Valente (2011) ao integrar essas tecnologias ao currículo escolar, as instituições podem abrir caminhos que potencializem novas formas de ensino e aprendizagem, garantindo que o estudante aprenda a lidar com o conhecimento.

Em meio a tantos recursos tecnológicos, podemos destacar a impressora 3D, que possibilita ao educador tornar concreto alguns objetos que até então só podiam ser observados virtualmente [Basniak e Liziero 2017]. Essa tecnologia vem ganhando espaço em sala de aula e auxiliando na compreensão do conteúdo trabalhado, visto que o manuseio e visualização dos objetos facilita o entendimento do mesmo [Silva, Siple e Figueiredo 2017].

Uma característica da impressora 3D, é a possibilidade de uso sem o conhecimento acerca da modelagem dos materiais. De acordo com Aguiar (2016) objetos educacionais podem facilmente ser encontrados em repositórios virtuais, bastando que o educador acesse e envie para que a impressora possa construir o material didático. Além disso, alguns softwares educacionais permitem que o professor crie modelos de forma simplificada e os converta para a impressão.

O software GeoGebra, por exemplo, contribui para a aprendizagem da geometria por meio de uma interface gráfica que permite a investigação de diversos conceitos matemáticos [Santos, Silva e Moura 2015]. Segundo Lemke, Siple e Figueiredo (2016) é possível integrar o GeoGebra à impressora 3D, possibilitando simular investigações antes restritas ao lápis e papel, contribuindo para tornar o processo mais significativo.

Um grande problema, porém, é a falta de uso desses recursos em sala de aula. Diversas escolas dispõem de laboratórios e de equipamentos tecnológicos de excelente qualidade, porém, o uso dessas tecnologias ainda é um desafio para um número considerável de professores na amplificação do seu trabalho em sala de aula [Rosa 2013].

Almeida e Valente (2011) destacam alguns fatores que contribuem para a falta de uso dessas tecnologias em sala de aula. Em conformidade com os autores, o rápido avanço das tecnologias e a falta de formação docente adequada para usá-las são um dos principais motivos para a desassociação das novas tecnologias ao currículo. Além disso, o professor não encontra apoio para desenvolver atividades que façam uso das tecnologias dentro do contexto de sala de aula, dificultando ainda mais o processo.

Partindo dessa problemática, este trabalho tem como objetivo mostrar uma possibilidade de uso das novas tecnologias em sala de aula, por meio de uma atividade desenvolvida junto ao professor de matemática em uma turma de $3^{\mathrm{a}}$ série do ensino médio, em uma escola pública da Paraíba.

A atividade consistiu no uso do software GeoGebra, associado à impressora 3D, para o ensino do conteúdo de geometria das coordenadas. Para isso, foi elaborado um plano de aula junto ao professor, buscando apresentá-lo formas de integrar as novas tecnologias em sua prática pedagógica e avaliar sua percepção após a conclusão da referida atividade. 


\section{Impressora 3D na Educação}

A impressora 3D no contexto educacional possibilita a construção de objetos sólidos que dificilmente são encontrados no mercado, proporcionando o desenvolvimento mediante o uso de softwares para a modelagem de objetos em computadores, para que logo em seguida possam ser impressos, proporcionando um ensino por meio de atividades práticas, mediante o manuseio de objetos reais [Basniak e Liziero 2017].

De acordo com Tramontano e Pereira Júnior (2015) a impressora 3D permite que os objetos sejam facilmente modificados, gerando novos modelos físicos. Os autores concluem que esses objetos podem permitir melhor visualização de detalhes, diferentemente dos físicos tradicionais. Para Blikstein (2013), os modelos físicos produzidos pela impressora 3D podem impactar na motivação dos estudantes, devido à grande qualidade das peças desenvolvidas, contribuindo para melhorar o processo de ensino.

Existem diversas situações em que a impressora 3D pode ser utilizada em detrimento da educação. Autores como Aguiar e Yonezawa (2014) destacam possibilidades para uso da ferramenta para beneficiar as aulas de física. Já Wen (2016), apresenta uma possibilidade de uso da impressora 3D para a produção de peças para o ensino da anatomia humana, facilitando a visualização de partes do corpo.

Outros estudos abordam possibilidades para o uso da impressora 3D no auxílio a deficientes visuais, em que peças táteis podem ser confeccionadas para auxílio ao ensino da física e matemática [Colpes 2014] ou a reprodução de peças artísticas, para que o estudante possa ter contato com as obras [Silva, Florindo e Machado 2017].

No ensino da matemática, em específico, alguns estudos apontam possibilidades para inserção dessa ferramenta. Lemke, Silva e Machado (2016) apresentam o uso da impressora 3D e o software GeoGebra para o desenvolvimento de objetos de aprendizagem, associando ao ensino de funções de várias variáveis.

$\mathrm{Na}$ mesma perspectiva, Basniak e Liziero (2017) desenvolveram um estudo que busca auxiliar professores na construção de modelos para o ensino da matemática, por acreditar que o uso da impressora 3D possibilita o desenvolvimento de materiais difíceis de desenvolver de outras formas.

Tantos estudos refletem diferentes possibilidades de uso da impressora 3D no contexto educacional, mostrando que diversos componentes curriculares podem contemplar o uso da impressora 3D. De modo geral, todos os estudos logram êxito em suas propostas, porém deixando evidente a necessidade de investimento material e de formação continuada para que os docentes adquiram independência na sua prática pedagógica.

\section{Software GeoGebra}

O GeoGebra é um software que permite aplicar a matemática em diversos níveis de ensino, contando com situações que envolvem geometria, álgebra, cálculo de planilhas, gráficos, probabilidade, estatística, dentre outros [GeoGebra 2020]. O software pode ser acessado gratuitamente, podendo o usuário realizar o download do material ou utilizá-lo on-line. 
De acordo com Santos, Silva e Moura (2015) o uso do GeoGebra permite desenvolver atividades não apenas com a parte geométrica, mas também algébrica, garantindo que o estudante compreenda as atividades de forma mais ampla. Além disso, o fato de ser um software interativo, torna a atividade mais dinâmica e significativa, outro fator que pode auxiliar na compreensão da atividade.

Lemke, Siple e Figueiredo (2016) também apontam para uma aprendizagem com maior significado ao usar o software GeoGebra. Para as autoras, a exploração do conteúdo a partir de papel e lápis constitui um ensino restrito, muitas vezes reduzido a reprodução automática de fórmulas. Com o GeoGebra, é possível simular representações, usando diferentes plataformas e abrangendo diferentes modalidades de ensino, seja presencial ou à distância.

\section{Metodologia}

Esse estudo adotou uma metodologia qualitativa, a partir de nuances da pesquisa-ação, apontada por Thiollent (2004) como um método de pesquisa que pode ser utilizado quando houver uma ação vinda dos sujeitos participantes no contexto observado. $\mathrm{Na}$ educação, constitui um método para resolver problemas no que diz respeito à formação das pessoas envolvidas, em que o pesquisador observa um problema e possíveis soluções para o mesmo [Elliot 1998].

Como instrumento para coleta de dados, foi realizada observação participante, durante a aplicação da atividade, seguido de entrevista com o professor responsável pela turma, em que o mesmo foi questionado a respeito das contribuições da impressora 3D e GeoGebra como aporte pedagógico.

Para o desenvolvimento da atividade proposta, foram analisadas as possibilidades de uso da impressora 3D como potencializadora do processo de ensino, para aplicá-la ao contexto de sala de aula. Para averiguar a viabilidade da aplicação, bem como compreender o processo, foi elaborado um plano de aula (Quadro 1) junto ao professor de matemática da instituição, responsável por ministrar a aula.

Segundo Góes et al. (2015) o plano de aula é importante pois uma aula excelente deve ser planejada. Nesse plano é fundamental que sejam observados diversos aspectos, visando a segurança do professor ao ministrar a aula, bem como para criar um ambiente adequado para repassar o conhecimento para os alunos. O plano deve ser definido como a antecipação dos assuntos e exercícios de uma aula.

Quadro 1. Recorte do plano de aula desenvolvido junto ao professor de matemática da instituição de ensino

Tema: Geometria das coordenadas.

Objetivo: Resolver situações-problema que envolvem relações métricas e trigonométricas no triângulo retângulo e no círculo.

Objetivos específicos:

Ao nível de conhecimento - Associar coordenadas cartesianas com figuras planas;

Ao nível de aplicação - Calcular distância entre pontos;

Ao nível de solução de problemas - Desenhar figuras planas a partir das coordenadas cartesianas de pontos no plano. 


\section{Conteúdo:}

$1^{\circ}$ estudando geometria analítica;

$2^{\circ}$ distância entre pontos;

$3^{\circ}$ condições de alinhamento de três pontos.

Desenvolvimento do tema: A partir de uma aula expositiva e dialogada será apresentado aos alunos os temas principais relacionados com as coordenadas cartesianas no plano. Será utilizado o GeoGebra para a visualização das coordenadas cartesianas de um ponto no plano e a impressora 3D para materializar um sólido, a partir do GeoGebra.

Recursos didáticos: Software GeoGebra, impressora 3D, projetor, computador.

Avaliação: avaliação formativa.

\subsection{Local da Pesquisa}

A escola em que o estudo foi desenvolvido oferece educação básica em tempo integral, atendendo às 3 séries do ensino médio. Além disso, oferta educação profissional integrada ao ensino médio, com os cursos técnicos em Informática e Mineração.

A instituição foi uma das contempladas pelo Pregão 003/2016, que previa a aquisição de material para laboratório de ciências em escolas do Estado da Paraíba. Entre os materiais disponibilizados, as escolas receberam uma impressora 3D modelo Winbo Mini para uso como material didático.

\subsection{Público-alvo}

Participaram da atividade desenvolvida, além do professor de matemática da instituição, alunos de uma turma da $3^{\text {a }}$ série do ensino médio. A escolha da referida turma ocorreu após observar que os mesmos não costumam participar de atividades que envolvam as novas tecnologias, de modo que a proposta desenvolvida poderia trazer um momento de inovação para os estudantes.

\section{Resultados e Discussão}

A atividade foi realizada no laboratório de informática, em que os estudantes foram conduzidos aos computadores para uso do software GeoGebra. Logo em seguida, o professor apresentou o software aos estudantes e junto a eles construiu os modelos propostos para a atividade em questão.

Ao término do processo, o modelo foi exportado para um software que permite realizar a conversão do modelo produzido no GeoGebra para que a impressora 3D possa construir. Dessa forma, os estudantes observaram a materialização da forma desenvolvida por eles durante o uso do GeoGebra e compreenderam o funcionamento da impressora $3 \mathrm{D}$. 
Concluindo a aula, cada estudante recebeu um modelo impresso (Figura 1), podendo realizar a manipulação e comparar com o modelo desenvolvido junto ao software GeoGebra.
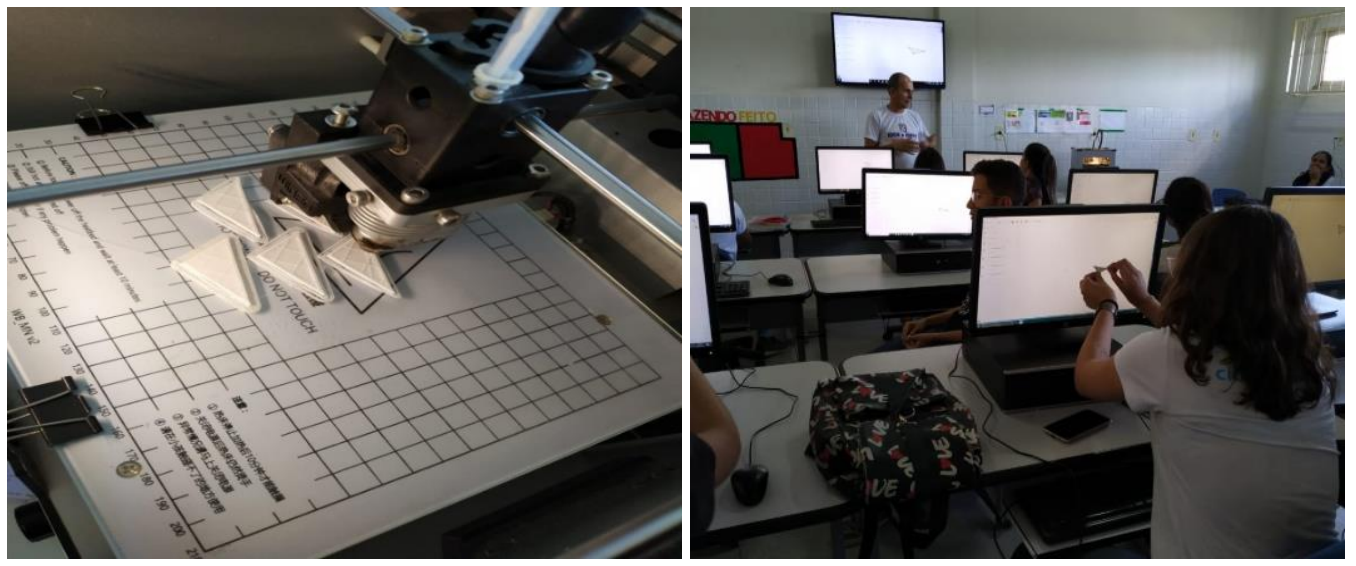

\section{Figura 1. (a) Impressão dos triângulos na impressora 3D; (b) Estudante comparando o modelo impresso com sua representação construída digitalmente}

Finalizada a atividade, foi realizada uma entrevista com o professor de matemática, visando identificar a percepção do mesmo com relação à atividade desenvolvida. A entrevista foi elaborada com base em Marconi e Lakatos (2003), que apontam as possibilidades da entrevista para eliminar as dúvidas do entrevistado, além de permitir obter dados mais relevantes do que outras formas de coleta de dados.

Foram elaboradas quatro perguntas, que versaram sobre a opinião do professor com relação a impressora 3D, o software GeoGebra em sala de aula, a possibilidade de contribuição da impressora 3D no processo, bem como o entendimento do professor quanto a outras possibilidades de uso da impressora 3D.

Abaixo, é possível observar as perguntas que constaram na entrevista $(\mathrm{P})$, bem como as respostas do professor $(\mathrm{R})$ :

$\mathrm{P}-$ Qual a sua opinião sobre a impressora 3D?

$\mathrm{R}$ - A impressora 3D é uma ferramenta poderosa que auxilia consideravelmente o processo de ensino-aprendizagem.

Lemke, Siple e Figueiredo (2016) abordam que um dos principais motivos que fazem da impressora 3D um objeto de inovação em sala é a possibilidade de construir diversos objetos. Entretanto, concluem que sem uma metodologia adequada, pode não vir a beneficiar o processo de ensino. Durante a atividade (Figura 2), ficou evidente o impacto inovador, tendo em vista a atenção e curiosidade dos estudantes durante o momento de produção e manipulação dos objetos 3D. 


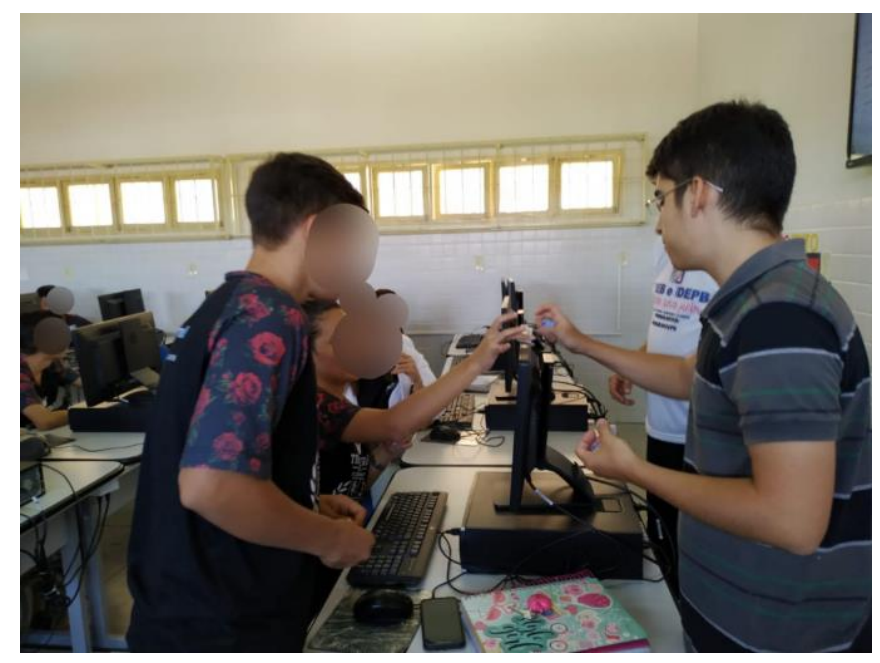

Figura 2. Manipulação dos objetos com os estudantes

$\mathrm{Na}$ pergunta seguinte, o professor foi questionado acerca do GeoGebra, associando ao software à facilidade no repasse do conteúdo.

$\mathrm{P}$ - Você considera que o GeoGebra facilitou no repasse do conteúdo a turma? Por quê?

$\mathrm{R}$ - Sim, a visualização em 2 e 3 dimensões facilita a visualização de relações geométricas e trigonométricas das figuras.

De fato, a impressora 3D e o GeoGebra permitem abordar de forma dinâmica as diversas versões de um objeto matemático [Lemke, Siple e Figueiredo 2016]. Durante a aula, essas ferramentas foram essenciais para que a visualização em 3D fosse possível, tendo em vista que até o momento da aula, os estudantes só haviam visualizado o conteúdo por meio do livro didático, que permite apenas visualização em 2D.

Em seguida, o professor relatou sua percepção quanto à contribuição da impressora 3D para que o estudante possa compreender o conteúdo.

$\mathrm{P}$ - Sobre a impressora 3D, você acha que ela contribuiu de alguma forma para o entendimento do conteúdo?

$\mathrm{R}$ - A impressora permite a materialização daquilo que foi desenhado.

Apesar de não ter ficado clara a opinião do professor com relação a este aspecto, Silva, Siple e Figueiredo (2017) destacam que a criação e o manuseio dos objetos podem facilitar a compreensão do assunto estudado. Afinal, a figura pode ser observada em seu plano completo, sem a necessidade de o estudante precisar imaginar o objeto estudado.

Finalizando a entrevista, foi realizado um momento reflexivo, em que o professor deveria apontar outras possibilidades de uso da impressora 3D, para uma posterior atividade usando esse recurso.

P - Você consegue imaginar outros conteúdos que sejam ensinados com auxílio da impressora 3D?

$\mathrm{R}$ - $\mathrm{O}$ estudo das cônicas pode ser facilitado com a impressora $3 \mathrm{D}$. 
Nicolletti e Figueiredo (2017) apontam a possibilidade para o uso da impressora 3D na produção de modelos para o estudo das cônicas. Entretanto, alertam que as representações 3D são mais difíceis de serem produzidas, pois a impressora 3D é uma tecnologia que exige um alto custo para a aquisição, de modo que não contempla todas as instituições de ensino.

\section{Considerações finais}

A atividade desenvolvida proporcionou identificar possibilidades para o uso da impressora no processo de ensino, principalmente no que diz respeito à facilidade em visualizar objetos que até então eram vistos apenas em $2 \mathrm{D}$. Além disso, permitiu levar ao contexto de sala de aula um momento de inovação, proporcionado pelo uso das tecnologias digitais.

Com o desenvolvimento do plano de aula, foi possível proporcionar ao professor um momento de reflexão acerca do desafio de inserir em sua prática pedagógica uma tecnologia digital até então sem muito uso na escola. Destaque para o domínio do docente, que mesmo não fazendo uso de recursos digitais com frequência, conseguiu lidar com fluência no manuseio de ferramentas como computador, projetor e impressora 3D.

O software GeoGebra mostrou-se relevante para transmitir o conteúdo de geometria das coordenadas, visto que permite construir os modelos, bem como realizar a manipulação através do software. Associado a isso, a impressora 3D garantiu a possibilidade de materializar a forma, facilitando ainda mais a visualização.

A entrevista com o professor permitiu perceber outras possibilidades de uso dessa ferramenta, não somente para a geometria das coordenadas, na matemática, mas também para outros conteúdos que compõem esse e outros componentes curriculares. Além disso, a partir do estudo da bibliografia, é possível constatar o uso da impressora 3D para o estudo da anatomia humana ou sua presença em cursos de design, por exemplo.

Apesar da boa prática realizada, ainda existem lacunas no que diz respeito a preparação de professores para uso dessa ferramenta, tendo em vista que a impressora 3D exige um conhecimento tecnológico adequado para seu uso. Torna-se necessário, portanto, maiores investimentos em formações continuadas, garantindo que essa e outras ferramentas não se tornem obsoletas pela falta de uso.

\section{Referências}

Aguiar, L. C. D. (2016). Um processo para utilizar a tecnologia de impressão 3D na construção de instrumentos didáticos para o ensino de ciências. Dissertação de Mestrado - Faculdade de Ciências, Bauru.

Aguiar, L. C. D. e Yonezawa, W. M. (2014). Construção de Instrumentos Didáticos com Impressoras 3D. In: Simpósio Nacional de Ensino de Ciência e Tecnologia.

Almeida, M. E. B. e Valente, J. A. (2011). Tecnologias e currículo: trajetórias convergentes ou divergentes?. São Paulo: Paulus.

Basniak, M. I. e Liziero, A. R. (2017). A IMPRESSORA 3D E NOVAS PERSPECTIVAS PARA O ENSINO: possibilidades permeadas pelo uso de materiais concretos. Revista Observatório, 3(4), p. 445-466. 
Blikstein, P. (2013). Digital Fabrication and 'Making' in Education The Democratization of Invention. In: Walter-Hermann, J., Büching, C. [Ed.]. FabLabs: Of Machines, Makers and Inventors. Bielefeld: Transcript Publishers. p. 261.

Bortolini, A., Gräbin, C., Gerhardt, L. M., Pereira, L. B., Scherer, M. S., Bersch, M. E. (2012). Reflexões sobre o uso das Tecnologias Digitais da Informação e da Comunicação no Processo Educativo. Revista Destaques Acadêmicos, 4(2), p. 141150 .

Colpes, K. M. (2014). Impressora de gráficos em alto-relevo para cegos: um facilitador no ensino da física e da matemática. Dissertação de Mestrado - Universidade Federal do Rio Grande do Sul, Porto Alegre.

Elliot, J. (1998). Recolocando a pesquisa-ação em seu lugar original e próprio. In: Geraldi, C. M. G.; Fiorentini, D.; Pereira, E. M. A. [Eds.]. Cartografias do trabalho docente: professor(a)-pesquisador(a). Campinas: Mercado de Letras. p. 336.

GeoGebra (2020). O que é o GeoGebra? Disponível em: 〈https://www.geogebra.org/>. Acesso em 23 de Abril de 2020.

Góes, F. S. N., Andrade, L. S., Côrrea, A. K., Mello e Souza, M. C. B., Clapis, M. J., Golçalves, M. F. C., Silva, M. A. I., Camargo, R. A. A. (2015). Plano de aula: apoio e fundamentos para prática docente. Ribeirão Preto: Escola Enfermagem Ribeirão Preto/USP.

Koch, M. Z. (2013). As Tecnologias no Cotidiano Escolar: Uma Ferramenta Facilitadora No Processo Ensino-Aprendizagem. Monografia de Especialização - Universidade Federal de Santa Maria, Sarandi.

Lemke, R., Siple, I. Z. e Figueiredo, E. B. (2016). OAs PARA O ENSINO DE CÁlCULO: POTENCIALIDADES DE TECNOLOGIAS 3D. Revista Novas Tecnologias na Educação, 14(1).

Marconi, M. e Lakatos, E. (2003). Fundamentos de metodologia científica. 5. ed. São Paulo: Editora Atlas.

Nicolletti, L. P. e Figueiredo, E. B. (2017). Cone de apolônio: história, matemática e material concreto. In: 27 SIC UDESC.

Rosa, R. (2013). Trabalho docente: dificuldades apontadas pelos professores no uso das tecnologias. Revista Encontro de Pesquisa em Educação, 1(1), p. 214-227.

Santos, A. S., Silva, J. J. e Moura, D. A. S. (2015). Tecnologia a favor da educação matemática: GeoGebra e suas aplicações. In: Encontro Mineiro de Educação Matemática.

Silva, J. R., Florindo, G. e Machado, V. (2017). Tecnologia 3D: Ferramenta para o ensino de Artes Visuais para pessoas com deficiência visual. In: Anais do XXVIII Simpósio Brasileiro de Informática na Educação (SBIE 2017).

Silva, S. M., Siple, I. Z. e Figueiredo, E. B. (2017). O uso da impressora 3D no ensino de matemática. In: 26 SIC UDESC.

Stinghen, R. (2016). Tecnologias na Educação: dificuldades encontradas para utilizá-la no ambiente escolar. Monografia de Especialização - Universidade Federal de Santa Catarina, Florianópolis. 
Thiollent, M. (2004). Metodologia da Pesquisa-Ação. 13. ed. São Paulo: Cortez.

Tramontano, M. e Pereira Júnior, A. (2015). Ressignificando o modelo físico: impressão 3D e ensino de projeto de arquitetura. In: XIX Congresso da Sociedade Iberoamericana de Gráfica Digital.

Wen, C. L. (2016). Homem Virtual (Ser Humano Virtual 3D): A Integração da Computação Gráfica, Impressão 3D e Realidade Virtual para Aprendizado de Anatomia, Fisiologia e Fisiopatologia. Revista de Graduação USP, 1(1), p. 7-16. 